\title{
Discrete time LQG controls with control dependent noise
}

\author{
John B. Moore ${ }^{\mathrm{a}, *}$, Xun Yu Zhou ${ }^{\mathrm{b}}$, Andrew E.B. Lim ${ }^{\mathrm{c}}$ \\ ${ }^{a}$ Department of Systems Engineering and Cooperative Research Center for Robust and Adaptive Systems, Research School of \\ Information Sciences and Engineering, Australian National University, Canberra, ACT 0200, Australia \\ ${ }^{\mathrm{b}}$ Department of Systems Engineering and Engineering Management, The Chinese University of Hong Kong, Shatin N.T., Hong Kong \\ ${ }^{\mathrm{c}}$ Department of Electrical \& Electronic Engineering, University of Melbourne, Parkville, Vic. 3052, Australia
}

Received 13 February 1998; received in revised form 26 August 1998; accepted 17 September 1998

\begin{abstract}
This paper presents some studies on partially observed linear quadratic Gaussian (LQG) models where the stochastic disturbances depend on both the states and the controls, and the measurements are bilinear in the noise and the states/controls. While the Separation Theorem of standard LQG design does not apply, suboptimal linear state estimate feedback controllers are derived based on certain linearizations. The controllers are useful for nonlinear stochastic systems where the linearized models include terms bilinear in the noise and states/controls and are significantly more accurate than if the bilinear terms are set to zero. The controllers are calculated by solving a generalized discrete time Riccati equation, which in turn has properties relating to well posedness of the associated LQG problem. (c) 1999 Elsevier Science B.V. All rights reserved.
\end{abstract}

\section{Introduction}

The classical linear quadratic Gaussian (LQG) control theory for stochastic linear systems assumes that the stochastic disturbances are additive and not control or state dependent [1-4]. Relaxing this assumption to allow state and control dependence in the noise terms leads to a broad class of stochastic models, which have applications for real-world control. For example, in a stock market the investments (controls) made by so-called "large investors" are going to affect fluctuations (disturbances) of the market. Working with models involving a bilinear noise dependence allows an improved approximation of the underlying nonlinear stochastic system.

Recently, linear quadratic regulator (LQR) theory has been generalized for a class of linear/bilinear stochastic systems in continuous time $[3,6]$. The asso-

\footnotetext{
*Corresponding author. e-mail: john.moore@anu.edu.au.
}

ciated optimal state feedback control laws are linear, being calculated by solving a so-called stochastic Riccati equation which specializes to the familiar conventional Riccati equation when the disturbances are independent of the states and controls. The stochastic Riccati equations are by no means as well understood as in the standard case, at least in the continuous time setting. There remains open questions concerning existence and uniqueness of the solutions of these equations. There is also an intriguing property that the control weighting matrix $R$ in a standard quadratic integral cost term need not be positive definite, even in the continuous time case.

What is the situation then for the partially observed case? To what extent does the standard LQG methodology [1] with its Separation Theorem apply? Can we achieve useful linear state estimate feedback laws?

In this paper the above questions are addressed for the discrete time case and some initial results are presented. The expectation is that since the 
models are bilinear in the state and the noise, as well as in the control and the noise, some of the virtues of the standard linear Gaussian theory will be lost. Certainly, even if the noise signals are Gaussian, the states and control signals will in general be non-Gaussian. Consequently, optimal (information) state estimators will be infinite dimensional, in general; see for example [5]. Even so, since a conditional linear minimum square error (LMSE) covariance state estimator is known for the models of interest, and is finite dimensional, it makes sense from an implementation point of view to work with such a state estimator and the resulting linear state estimate feedback law, even if such a law is suboptimal.

The conditional LMSE filter has the structure of a Kalman filter, see [1], but with a Kalman gain which is state estimate and control dependent. Likewise, the quadratic state cost when expressed in terms of state estimates instead of true states is nonlinear. Appropriate linearizations of the filter equations and cost terms, neglecting higher order terms but allowing terms bilinear in the noise and controls/state estimates in the filter, allows application of a discrete-time analogy of the recently studied LQR theory in [3]. This leads to an 'optimal' linear state estimate feedback law under assumptions of negligible higher order terms. In practise, this law has some degree of sub-optimality because the neglected higher-order terms may be significant. However, the neglected terms do not include terms bilinear in the innovations (prediction errors) and the state estimates/controls, so there is a chance for improved performance over the standard LQG approach which neglects these terms as well as higher-order terms.

The paper is organized as follows. In Section 2 an optimal feedback controller is derived for a completely observed discrete time, linear quadratic regulators (LQR) with state- and control-dependent noise. As in the standard case, solving a discrete time Riccati equation is a key step in calculating the optimal controller. In fact, the associated Riccati equation is a generalization of the standard discrete time Riccati equation. The existence properties of this equation and its relationship to the well posedness of the control problem is discussed in Section 3. Section 4 is concerned with an approximate Kalman filter for the partially observed LQG model. Finally, suboptimal linear state estimate feedback laws are obtained in Section 5 by combining the results in Sections 2 and 4.

\section{Discrete time LQR results}

In this section, we derive parallel results to those of [3], but in discrete time rather than continuous time. These will be useful in a later section. The results in this section are also of interest on their own right, as discrete time algorithms are useful in practice.

Consider the discrete time stochastic signal model

$$
x_{k+1}=\left(A_{k}+w_{k}^{A} \Delta A_{k}\right) x_{k}+\left(B_{k}+w_{k}^{B} \Delta B_{k}\right) u_{k}+w_{k},
$$

where $x_{k} \in \mathbb{R}^{n}$ is the state, $u_{k} \in \mathbb{R}^{m}$ is the control, and $w_{k}^{A}, w_{k}^{B} \in \mathbb{R}$ are noise terms, assumed here to be martingale increments on $\mathscr{G}_{k-1}$, where $\mathscr{G}_{k-1}$ is the $\sigma$-algebra generated by past noise terms up to $w_{k-1}^{A}, w_{k-1}^{B}, w_{k-1}$. Thus $x_{k}$ is measurable with respect to $\mathscr{G}_{k}$ and

$E\left[w_{k-1}^{A} \mid \mathscr{G}_{k-1}\right]=E\left[w_{k-1}^{B} \mid \mathscr{G}_{k-1}\right]=E\left[w_{k-1} \mid \mathscr{G}_{k-1}\right]=0$.

The covariances are assumed to be

$E\left[\left(w_{k-1}^{A}\right)^{2} \mid \mathscr{G}_{k-1}\right]=E\left[\left(w_{k-1}^{B}\right)^{2} \mid \mathscr{G}_{k-1}\right]=1$,

$E\left[w_{k} w_{k}^{\prime} \mid \mathscr{G}_{k-1}\right]=Q_{k}$

and

$E\left[w_{k-1}^{A} w_{k-1}^{B} \mid \mathscr{G}_{k-1}\right]=\rho_{k}^{A B}$,

$E\left[w_{k-1} w_{k-1}^{A} \mid \mathscr{G}_{k-1}\right]=\rho_{k}^{A}$,

$E\left[w_{k-1} w_{k-1}^{B} \mid \mathscr{G}_{k-1}\right]=\rho_{k}^{B}$.

Generalizations of the dependent noise terms $w_{k}^{A} \Delta A_{k}$ and $w_{k}^{B} \Delta B_{k}$ to the case of non-scalar noise is immediate by working with terms $\sum_{i=1}^{N} w_{k}^{A^{i}} \Delta A_{k}^{i}$ and $\sum_{i=1}^{N} w_{k}^{B^{i}} \Delta B_{k}^{i}$.

It should be noted that the time-varying versions of Eq. (1) result from linearizations of nonlinear stochastic models of the form $x_{k+1}=f\left(x_{k}, u_{k}, w_{k}\right)$. Linearizations which set the bilinear terms in the noise to zero result in the standard stochastic models.

The performance index of the problem is given by the standard quadratic sum cost

$$
\begin{gathered}
J_{T}=E\left\{\sum_{k=0}^{T-1}\left(x_{k}^{\prime} Q_{k}^{c} x_{k}+u_{k}^{\prime} R_{k+1}^{c} u_{k}\right)\right. \\
\left.+x_{T}^{\prime} Q_{T}^{c} x_{T}-x_{0}^{\prime} Q_{0}^{c} x_{0}\right\} .
\end{gathered}
$$


In this model, all the $A_{k}, \Delta A_{k}$, etc. are (deterministic) matrices with appropriate dimensions, $Q_{k}^{c}$ and $Q$ are non-negative definite matrices, and $R_{k}^{c}$ are symmetric matrices (could be indefinite, as in standard discrete time LQR theory).

Let us solve the above stochastic optimal control problem in two different cases. The results derived below will be applied in Section 4 for partially observed models.

Case I: $\rho_{k}^{A}=\rho_{k}^{B}=0$.

Let us consider first the case when $w_{k} \perp w_{k}^{A}, w_{k}^{B}$, so that $\rho_{k}^{A}=\rho_{k}^{B}=0$. In this case, we are going to show that the optimal control takes the form

$u_{k}=K_{k}^{c} x_{k}$,

where

$K_{k}^{c}=-\left(\Omega_{k+1}^{c}\right)^{-1} L_{k+1}^{c}$,

$L_{k+1}^{c}=B_{k}^{\prime} S_{k+1} A_{k}+\rho_{k}^{A B} \Delta B_{k}^{\prime} S_{k+1} \Delta A_{k}$,

$\Omega_{k+1}^{c}=B_{k}^{\prime} S_{k+1} B_{k}+\Delta B_{k}^{\prime} S_{k+1} \Delta B_{k}+R_{k+1}^{c}$.

Here, $S_{k}$ is the solution of a backward matrix Riccati equation

$$
\begin{aligned}
S_{k}= & A_{k}^{\prime} S_{k+1} A_{k}-L_{k+1}^{c \prime}\left(\Omega_{k+1}^{c}\right)^{-1} L_{k+1}^{c} \\
& +\left(Q_{k}^{c}+\Delta A_{k}^{\prime} S_{k+1} \Delta A_{k}\right), \\
S_{T}= & Q_{T}^{c} .
\end{aligned}
$$

In fact, assuming the existence of the solution $S_{k}$ of Eq. (4), the control law (3)-(4) is seen to be optimal by completion of the square arguments as follows. First, note that

$$
\sum_{k=0}^{T-1}\left(x_{k}^{\prime} S_{k} x_{k}-x_{k+1}^{\prime} S_{k+1} x_{k+1}\right)=x_{0}^{\prime} S_{0} x_{0}-x_{T}^{\prime} S_{T} x_{T}
$$

and

$$
\begin{aligned}
\left(u_{k}-\right. & \left.K_{k}^{c} x_{k}\right)^{\prime} \Omega_{k+1}^{c}\left(u_{k}-K_{k}^{c} x_{k}\right) \\
= & u_{k}^{\prime} \Omega_{k+1}^{c} u_{k}+2 x_{k}^{\prime} L_{k+1}^{c \prime} u_{k} \\
& +x_{k}^{\prime} L_{k+1}^{c \prime}\left(\Omega_{k+1}^{c}\right)^{-1} L_{k+1}^{c} x_{k} \\
= & u_{k}^{\prime}\left(R_{k+1}^{c}+B_{k}^{\prime} S_{k+1} B_{k}+\Delta B_{k}^{\prime} S_{k+1} \Delta B_{k}\right) u_{k} \\
& +2 x_{k}^{\prime}\left(A_{k}^{\prime} S_{k+1} B_{k}+\rho_{k}^{A B} \Delta A_{k}^{\prime} S_{k+1} \Delta B_{k}\right) u_{k} \\
& +x_{k}^{\prime} L_{k+1}^{c \prime}\left(\Omega_{k+1}^{c}\right)^{-1} L_{k+1}^{c} x_{k} .
\end{aligned}
$$

Hence $J_{T}$ can be re-organized by using Eqs. (4)-(6) and eliminating $Q_{k}^{c}, R_{k+1}^{c}$ to yield

$$
\begin{aligned}
J_{T}=E\left\{\sum_{k=0}^{T-1}\left(u_{k}-K_{k}^{c} x_{k}\right)^{\prime} \Omega_{k+1}^{c}\left(u_{k}-K_{k}^{c} x_{k}\right)\right. \\
\left.+x_{0}^{\prime}\left(S_{0}-Q_{0}^{c}\right) x_{0}\right\} \\
+E\left\{\sum _ { k = 0 } ^ { T - 1 } \left[x_{k+1}^{\prime} S_{k+1} x_{k+1}-x_{k}^{\prime} A_{k}^{\prime} S_{k+1} A_{k} x_{k}\right.\right. \\
\quad-x_{k}^{\prime} \Delta A_{k}^{\prime} S_{k+1} \Delta A_{k} x_{k}-2 x_{k}^{\prime} L_{k+1}^{c \prime} u_{k} \\
\left.\left.\quad-u_{k}^{\prime}\left(B_{k}^{\prime} S_{k+1} B_{k}+\Delta B_{k}^{\prime} S_{k+1} \Delta B_{k}\right) u_{k}\right]\right\} .
\end{aligned}
$$

Substituting for $x_{k+1}$ from Eq. (1), and from $L_{k+1}^{c}$, the third term simplifies as $\sum_{k=0}^{T-1} \operatorname{tr}\left(S_{k+1} Q_{k}\right)$. That is,

$$
\begin{array}{r}
J_{T}=E\left\{\sum_{k=0}^{T-1}\left(u_{k}-K_{k}^{c} x_{k}\right)^{\prime} \Omega_{k+1}^{c}\left(u_{k}-K_{k}^{c} x_{k}\right)\right\} \\
+\sum_{k=0}^{T-1} \operatorname{tr}\left(S_{k+1} Q_{k}\right)+x_{0}^{\prime}\left(S_{0}-Q_{0}^{c}\right) x_{0} .
\end{array}
$$

Therefore Eqs. (1) and (2) are well posed if $\Omega_{k+1}^{c}$ is positive definite. In this case, the control law (2) $-(4)$ is the unique optimal control which achieves a minimum cost

$J_{T}(\min )=\sum_{k=0}^{T-1} \operatorname{tr}\left(S_{k+1} Q_{k}\right)+x_{0}^{\prime}\left(S_{0}-Q_{0}^{c}\right) x_{0}$.

However, we have assumed that $\Omega_{k+1}^{c}$ is strictly positive definite for every $k$. In fact, by defining $K_{k}^{c}$ as a solution of the equation $\Omega_{K+1}^{c} K_{k}^{c}=L_{k+1}^{c}$, and using the concept of pseudo-inverses, the above results can be shown to hold for the case when $\Omega_{k+1}^{c}$ is positive semi-definite. That is, the LQR problem (1)-(2) is well posed if and only if $\Omega_{k+1}^{c}$ is positive semi-definite. In Section 3, we shall address this issue of existence of solutions in more detail. In particular, we shall examine the effect of the bilinear terms on the solution of the Riccati equation (4), and the well posedness of Eqs. (1) and (2).

In continuous time LQR theory, a standard assumption is that the control weighting matrix $R$ is strictly positive definite. This is necessary for the problem to be well posed. Recent results by Chen et al. [3] for the continuous time problem show that $R$ can have 
negative eigenvalues if the diffusion term in the system equations depends on the control. It is interesting to note therefore that in the discrete time problem, the control weighting matrices $R_{k}$ can have negative eigenvalues and the problem remain well posed, even if the bilinear terms $\Delta A_{k}$ and $\Delta B_{k}$ are all zero! That is, $\Omega_{k+1}^{c}$ can be positive semi-definite, even if some or all the $R_{k}$ matrices have negative eigenvalues, and $\Delta A$ and $\Delta B$ are zero. Of course, if $\Delta A$ or $\Delta B$ are non-zero, then $R_{k}$ can be 'more' negative-definite and the problem still remain well posed.

Case II: $\rho_{k}^{A} \neq 0, \rho_{k}^{B} \neq 0$.

In the event that $w_{k}$ and $\left(w_{k}^{A}, w_{k}^{B}\right)$ are correlated so that $\rho_{k}^{A}, \rho_{k}^{B} \neq 0$, then the optimal control requires not only the state feedback term as in Eq. (3) but also an external input as

$u_{k}=K_{k}^{c} x_{k}+b_{k}$,

where $b_{k}$ is calculated by linear backward recursions as now described.

Without loss of generality, assume $\bar{x}_{0}:=E\left[x_{0}\right]=0$, and define

$$
\begin{aligned}
& \alpha_{k}^{A}:=S_{k+1} \Delta A_{k} \rho_{k}^{A}, \quad \alpha_{k}^{B}:=S_{k+1} \Delta B_{k} \rho_{k}^{B}, \\
& \beta_{k}^{A}:=\Omega_{k+1} K_{k}, \quad \beta_{k}^{B}:=\Omega_{k+1} .
\end{aligned}
$$

Going through similar calculations as in Eq. (7), one ends up with the following additional cost term involving $b_{k}, \rho_{k}^{A}, \rho_{k}^{B}$ :

$J^{\text {odd }}=\sum_{k=0}^{T-1}\left(\left(\alpha_{k}^{A}-b_{k}^{\prime} \beta_{k}^{A}\right) \bar{x}_{k}+\left(\alpha_{k}^{B}-b_{k}^{\prime} \beta_{k}^{B}\right) \bar{u}_{k}\right)$.

Here $\bar{x}_{k}:=E\left[x_{k}\right], \quad \bar{u}_{k}:=E\left[u_{k}\right]$ which satisfy, from taking expectations in Eq. (1), $\bar{x}_{k+1}=A_{k} \bar{x}_{k}+$ $B_{k} \bar{u}_{k}$. To see that $J^{\text {odd }}=0$ can hold by a suitable $b_{k}$ selection, first substitute $\bar{x}_{0}=0, \quad \bar{x}_{1}=A_{0} \bar{x}_{0}+$ $B_{0} \bar{u}_{0}=B_{0} \bar{u}_{0}, \bar{x}_{2}=A_{1} B_{0} \bar{x}_{0}+B_{1} \bar{u}_{1}$, etc. Then, Eq. (12) can be re-organized as a matrix equation

$\left[\begin{array}{llll}1 & 1 & \cdots & 1\end{array}\right]\left[\begin{array}{cccc}\times & 0 & \ldots & 0 \\ \times & \times & & 0 \\ \vdots & & \ddots & \\ \times & \times & \ldots & \times\end{array}\right]\left[\begin{array}{c}\bar{u}_{0} \\ \bar{u}_{1} \\ \vdots \\ \bar{u}_{T}\end{array}\right]=0$.

Denoting the lower triangular matrix as $L$ and the row vector $\left[\begin{array}{llll}1 & 1 & \cdots & 1\end{array}\right]$ as $\underline{\mathbf{1}}^{\prime}$, then this holds if $\underline{\mathbf{1}}^{\prime} L=0$; that is, the sum of the columns of $L$ is zero. Starting with the last column allows calculation of $b_{T}$, and then the second last column allows calculation of
$b_{T-1}$ in terms of $b_{T}$. Proceeding, allows calculation of $b_{k}$ in terms of $b_{k+1}, \ldots, b_{T}$.

Thus, using a backwards recursion, $\left\{b_{k}\right\}$ is calculated by solving successively (with forward substitutions)

$$
\begin{aligned}
& \alpha_{T}^{B}-b_{T} \beta^{B}=0, \\
& \left(\alpha_{T}^{A}-b_{T} \beta_{T}^{A}\right) B_{T-1}+\left(\alpha_{T-1}^{B}-b_{T-1} \beta_{T-1}^{B}\right)=0, \\
& \quad \quad \vdots \\
& \quad\left(\alpha_{T}^{A}-b_{T} \beta_{T}^{A}\right)\left(A_{T-1} A_{T-2} \cdots A_{1} B_{0}\right) \\
& \quad+\left(\alpha_{T-1}^{A}-b_{T-1} \beta_{T-1}^{A}\right)\left(A_{T-2} \cdots A_{1} B_{0}\right)+\cdots \\
& \quad+\left(\alpha_{0}-b_{0} \beta_{0}^{B}\right)=0,
\end{aligned}
$$

for $b_{T}, b_{T-1}, b_{T-2}, \ldots, b_{0}$. The equations have the form $\mathscr{L} b=d$ where $\mathscr{L}$ is lower triangular and $b^{\prime}=\left[\begin{array}{llll}b_{T} & b_{T-1} & \cdots & b_{0}\end{array}\right]$, which is readily solved for $b$.

\section{Discrete time Riccati equation}

In the continuous time $\mathrm{LQR}$ problem, a standard assumption is that the control weighting matrix $R(t)$ is strictly positive definite. In the paper by Chen et al. [3], it is shown that for full observation stochastic LQR problems with control-dependent diffusion terms, this assumption is not necessary. In fact, they derive necessary and sufficient conditions for the solvability of the associated Riccati equation and show that these conditions can be satisfied (and the associated LQR problem well posed) by control weighting matrices with negative eigenvalues. In this section, we examine the effect of the terms $\Delta A_{k}$ and $\Delta B_{k}$ on the well posedness of the LQR problem (1)-(2).

Recall that the LQR problem (1)-(2) is well posed if and only if $\Omega_{k}^{c} \geqslant 0$ for every $k$. Note once again that it is possible for the standard LQR problem (i.e. $\Delta A_{k}=0$ and $\left.\Delta B_{k}=0\right)$ to be well posed with either $Q_{k}<0$ or $R_{k}<0$ (but obviously not both). We show in this section that if $\Delta A_{k} \neq 0$ or $\Delta B_{k} \neq 0$, then $Q_{k}$ and $R_{k}$ can be made 'more negative'. That is, we can replace $Q_{k}$ by $\bar{Q}_{k} \leqslant Q_{k}$ and $R_{k}$ by $\bar{R}_{k} \leqslant R_{k}$ and with the associated problem still remaining well posed. Bounds on the allowable decrease are also derived for certain special cases.

Before doing this however, we need to introduce some notation. Let $\mathscr{K}=\left\{\left(S_{0}, \ldots, S_{T}\right) \mid S_{j} \in \mathbb{R}^{n \times n}\right.$, symmetric $\}, \quad \mathscr{Q}=\left\{\left(Q_{0}, \ldots, Q_{T}\right) \mid Q_{j} \in \mathbb{R}^{n \times n}\right.$, symmetric $\}$ and $\mathscr{P}=\left\{\left(R_{1}, \ldots, R_{T}\right) \mid R_{j} \in \mathbb{R}^{m \times m}\right.$, symmetric $\}$. Given a sequence $\bar{R}^{c}=\left(\bar{R}_{1}^{c}, \ldots, \bar{R}_{T}^{c}\right) \in \mathscr{P}$ of control 
weights and $\bar{Q}^{c}=\left(\bar{Q}_{0}^{c}, \ldots, \bar{Q}_{T}^{c}\right) \in \mathscr{Q}$ of state weights, the standard discrete time Riccati equation

$$
\begin{aligned}
S_{k}= & A_{k}^{\prime} S_{k+1} A_{k}-A_{k}^{\prime} S_{k+1} B_{k}\left(\bar{R}_{k+1}^{c}\right. \\
& \left.+B_{k}^{\prime} S_{k+1} B_{k}\right)^{-1} B_{k}^{\prime} S_{k+1} A_{k}+\bar{Q}_{k}^{c}, \\
S_{T}= & \bar{Q}_{T}^{c},
\end{aligned}
$$

gives rise to a sequence $\left(S_{0}, \ldots, S_{T}\right) \in \mathscr{K}$. Hence, we can define a mapping $\psi: \mathscr{P} \times \mathscr{Z} \rightarrow \mathscr{K}$ which maps a sequence of control weights $\bar{R}^{c}=\left(\bar{R}_{1}^{c}, \ldots, \bar{R}_{T}^{c}\right) \in \mathscr{P}$ and state weights $\bar{Q}^{c}=\left(\bar{Q}_{0}^{c}, \ldots, \bar{Q}_{T}^{c}\right) \in \mathscr{Q}$ to the solution $\psi\left(\bar{Q}^{c}, \bar{R}^{c}\right)=\left(\psi_{0}\left(\bar{Q}^{c}, \bar{R}^{c}\right), \ldots, \psi_{T}\left(\bar{Q}^{c}, \bar{R}^{c}\right)\right) \in \mathscr{K}$ of Eq. (14).

Suppose now that $\bar{Q}^{c}=Q^{c} \in \mathscr{Q}$ is given (and fixed) while $\bar{R}^{c}$ is the variable. In this case, we shall write $\psi\left(Q^{c}, \bar{R}^{c}\right)$ simply as $\psi\left(\bar{R}^{c}\right)$. It follows that the associated (standard) LQR problem is solvable if and only if

$\bar{R}_{k+1}^{c}+B_{k}^{\prime} \psi_{k+1}\left(\bar{R}^{c}\right) B_{k} \geqslant 0$.

We begin by examining the case $\Delta A_{k}=0$. Before stating our main results, we note the following.

Lemma 3.1. Let $\Delta A_{k}=0$ and $\Delta B_{k} \neq 0$. Let $\left(Q_{k}^{c}, R_{k}^{c}\right)$ be given. Then Eqs. (1) and (2) are well posed if and only if there exists $\bar{R}_{k}^{c}$ such that

$R_{k+1}^{c}+\Delta B_{k}^{\prime} \psi_{k+1}\left(\bar{R}^{c}\right) \Delta B_{k}=\bar{R}_{k+1}^{c}$,

where $\left(Q_{k}^{c}, \bar{R}_{k}^{c}\right)$ satisfy Eqs. (14) and (15).

Proof. Obvious.

Remark 3.1. In the continuous time case [3], the existence of a solution to the Riccati equation that satisfies a condition similar to the one in Lemma 3.1 is sufficient for well posedness of the LQR problem. However, it is not necessary for well posedness. This arises in the continuous time case because the Riccati equation may not have a solution. On the other hand, the Riccati equation associated with the discrete time problem always has a solution if pseudo-inverses are allowed.

Lemma 3.2. If Eqs. (1) and (2) corresponding to $\left(Q_{k}^{c}, R_{k}^{c}\right)$ are well posed, then Eqs. (1) and (2) with $\left(Q_{k}^{c}, \tilde{R}_{k}^{c}\right)$ are well posed for all $\tilde{R}_{k}^{c} \geqslant R_{k}^{c}$.
Proof. Let $\Delta=\tilde{R}_{k}^{c}-R_{k}^{c} \geqslant 0$. Then for every feasible $u_{k}$, we have

$$
\begin{aligned}
& J_{T}=E\left\{\sum_{k=0}^{T-1}\left(x_{k}^{\prime} Q_{k}^{c} x_{k}+u_{k}^{\prime} \tilde{R}_{k+1}^{c} u_{k}\right)\right. \\
& \left.+x_{T}^{\prime} Q_{T}^{c} x_{T}-x_{0}^{\prime} Q_{0}^{c} x_{0}\right\} \\
& =E\left\{\sum_{k=0}^{T-1}\left(x_{k}^{\prime} Q_{k}^{c} x_{k}+u_{k}^{\prime}\left(R_{k+1}^{c}+\Delta_{k+1}\right) u_{k}\right)\right. \\
& \left.+x_{T}^{\prime} Q_{T}^{c} x_{T}-x_{0}^{\prime} Q_{0}^{c} x_{0}\right\} \\
& =E\left\{\sum_{k=0}^{T-1}\left(x_{k}^{\prime} Q_{k}^{c} x_{k}+u_{k}^{\prime} R_{k+1}^{c} u_{k}\right)\right. \\
& \left.+x_{T}^{\prime} Q_{T}^{c} x_{T}-x_{0}^{\prime} Q_{0}^{c} x_{0}\right\} \\
& +E\left\{\sum_{k=0}^{T-1} u_{k}^{\prime} \Delta_{k+1} u_{k}\right\} \\
& \geqslant E\left\{\sum_{k=0}^{T-1}\left(x_{k}^{\prime} Q_{k}^{c} x_{k}+u_{k}^{\prime} R_{k+1}^{c} u_{k}\right)+x_{T}^{\prime} Q_{T}^{c} x_{T}\right. \\
& \left.-x_{0}^{\prime} Q_{0}^{c} x_{0}\right\} \\
& >0
\end{aligned}
$$

from which the result follows.

We are now in the position to state our main result regarding the influence of the term $\Delta B_{k}$ on the problem (1)-(2).

Theorem 3.1. Let $\left(Q_{k}^{c}, R_{k}^{c}\right)$ be given. If $\Delta A_{k}=0$, then the problem (1)-(2) corresponding to $\left(Q_{k}^{c}, R_{k}^{c}\right)$ is well posed if and only if

$R_{k+1}^{c} \geqslant \bar{R}_{k+1}^{c}-\Delta B_{k}^{\prime} \psi_{k+1}\left(\bar{R}^{c}\right) \Delta B_{k}$

for some $\bar{R}_{k}^{c}$ such that $\left(\bar{Q}_{k}^{c}=Q_{k}^{c}, \bar{R}_{k}^{c}\right)$ satisfies Eqs. (14) and (15).

Proof. Suppose that Eqs. (1) and (2) is well posed for $\left(Q_{k}^{c}, R_{k}^{c}\right)$. Then by Lemma 3.1 , there exists $\bar{R}_{k}$ such that

$R_{k+1}^{c}+\Delta B_{k}^{\prime} \psi_{k+1}\left(\bar{R}^{c}\right) \Delta B_{k}=\bar{R}_{k+1}^{c}$

and $\left(Q_{k}^{c}, \bar{R}_{k}^{c}\right)$ satisfy Eqs. (14) and (15). This implies Eq. (17). 
Conversely, suppose that Eq. (17) holds for some $\bar{R}_{k}^{c}$ such that $\left(Q_{k}^{c}, \bar{R}_{k}^{c}\right)$ satisfies Eqs. (14) and (15). Then there exists some $\tilde{R}_{k}^{c}$ such that

$R_{k+1}^{c} \geqslant \tilde{R}_{k+1}^{c}=\bar{R}_{k+1}^{c}-\Delta B_{k}^{\prime} \psi_{k+1}\left(\bar{R}^{c}\right) \Delta B_{k}$.

By Lemma 3.1, the problem (1)-(2) associated with $\left(Q_{k}^{c}, \tilde{R}_{k}^{c}\right)$ is well posed. Since $R_{k}^{c} \geqslant \tilde{R}_{k}^{c}$, it follows from Lemma 3.2 that Eqs. (1) and (2) with $\left(Q_{k}^{c}, R_{k}^{c}\right)$ are well posed.

Note in particular that if $\Delta B_{k} \neq 0$, then the control weighting matrices $\tilde{R}_{k}^{c}$ can be made 'more negative' and the problem (1) $-(2)$ still remains well posed. That is, if $\Delta B_{k} \neq 0$, the matrices $\tilde{R}_{k}^{c}$ can be replaced by matrices $R^{c}$ such that $R_{k}^{c} \leqslant \bar{R}_{k}^{c}$, and the problem (1)(2) still remains well posed. The bound on this change is given by Eq. (17).

In the analysis above, we have assumed that $\bar{Q}^{c}$ is given and fixed. In fact, if we define the inner product $\langle\cdot, \cdot\rangle_{\mathscr{Q}}: \mathscr{Q} \times \mathscr{Q} \rightarrow \mathbb{R}$ on $\mathscr{Q}$ by

$$
\left\langle Q^{1}, Q^{2}\right\rangle=\sum_{k=0}^{T} \operatorname{tr}\left(Q_{k}^{1} \cdot Q_{k}^{2}\right)
$$

and $\langle\cdot, \cdot\rangle: \mathscr{K} \times \mathscr{K} \rightarrow \mathbb{R}$ similarly, then it is easily shown that $\psi_{k+1}\left(\bar{Q}^{c}, \bar{R}^{c}\right)$ is continuous with respect to $\bar{Q}^{c}$. It follows then that $\bar{Q}_{k}^{c}$ can be made 'more negative' if $\Delta A_{k}=0$ and $\Delta B_{k} \neq 0$. The allowable bounds on this change is still an open question.

Consider now the case when $\Delta A_{k} \neq 0$ but $\Delta B_{k}=0$. Let $\bar{R}^{c}=R^{c} \in \mathscr{P}$ be fixed. Let $\psi: \mathscr{Q} \rightarrow \mathscr{K}$ be a mapping such that $\psi\left(\bar{Q}^{c}\right)=\psi\left(\bar{Q}^{c}, R^{c}\right)$ is the solution of the standard discrete time Riccati equation

$$
\begin{aligned}
S_{k}= & A_{k}^{\prime} S_{k+1} A_{k}-A_{k}^{\prime} S_{k+1} B_{k}\left(R_{k+1}^{c}+B_{k}^{\prime} S_{k+1} B_{k}\right)^{-1} \\
& B_{k}^{\prime} S_{k+1} A_{k}+\bar{Q}_{k}^{c}, \\
S_{T}= & \bar{Q}_{T}^{c} .
\end{aligned}
$$

In this case, the associated (standard) LQR problem is solvable if and only if

$R_{k+1}^{c}+B_{k}^{\prime} \psi_{k+1}\left(\bar{Q}^{c}\right) B_{k} \geqslant 0$.

In much the same way as the case $\Delta A_{k}=0, \Delta B_{k} \neq 0$, the following result can be shown.

Theorem 3.2. Let $\left(Q_{k}^{c}, R_{k}^{c}\right)$ be given. If $\Delta B_{k}=0$, then the problem (1)-(2) corresponding to $\left(Q_{k}^{c}, R_{k}^{c}\right)$ is well posed if and only if

$Q_{k}^{c} \geqslant \bar{Q}_{k}^{c}-\Delta A_{k}^{\prime} \psi_{k+1}\left(\bar{Q}^{c}\right) \Delta A_{k}$ for some $\bar{Q}_{k}^{c}$ such that $\left(R_{k}^{c}, \bar{Q}_{k}^{c}\right)$ satisfies Eqs. (20) and (21).

As in the case of Theorem 3.1, Theorem 3.2 shows how much 'more negative' the matrices $\bar{Q}_{k}^{c}$ can be made when $\Delta A_{k} \neq 0$ and $\Delta B_{k}=0$. Furthermore, if we define an inner product $\langle\cdot, \cdot\rangle_{\mathscr{P}}: \mathscr{P} \times \mathscr{P} \rightarrow \mathbb{R}$ on $\mathscr{P}$ as we have for $\mathscr{Q}$ (see Eq. (19)), it is easily shown that $\psi_{k+1}\left(\bar{Q}^{c}, \bar{R}^{c}\right)$ is continuous with respect to $\bar{R}^{c}$. Therefore, if $\Delta A_{k} \neq 0$ and $\Delta B_{K}=0, \bar{R}_{k}^{c}$ can be made 'more negative'. The allowable bounds on this change is still an open question. Similarly, the effect of both $\Delta A_{k} \neq 0$ and $\Delta B_{k} \neq 0$ is still unresolved.

\section{State estimation}

In this section, we first define a partially observed signal model. Next, we apply the known Kalman filter theory to yield a linear minimum variance state estimator, which is then linearized further so that the filter is linear in the states and control, and bilinear in the innovations (prediction errors) and the states/ controls.

Consider the following partially observed model:

$$
\begin{aligned}
& x_{k+1}=\left(A_{k}+w_{k}^{A} \Delta A_{k}\right) x_{k}+\left(B_{k}+w_{k}^{B} \Delta B_{k}\right) u_{k}+w_{k}, \\
& y_{k}=\left(C_{k}+w_{k}^{c} \Delta C_{k}\right) x_{k}+v_{k},
\end{aligned}
$$

where $y_{k} \in \mathbb{R}^{p}$. Here $w_{k}^{c}, v_{k}$ are martingale increments, each orthogonal to $w_{k}^{\mathrm{A}}, w_{k}^{\mathrm{B}}, w_{k}$, and $E\left[v_{k} v_{k}^{\prime}\right]=R_{k}$.

Linear conditional minimum variance state estimator: Applying standard filtering results [1] yields the estimator

$$
\begin{aligned}
& \hat{x}_{k+1}=A_{k} \hat{x}_{k}+B_{k} u_{k}+K_{k}\left(\hat{x}_{k}, u_{k}\right) v_{k}, \\
& v_{k}=y_{k}-C_{k} \hat{x}_{k},
\end{aligned}
$$

where the gain $K_{k}\left(\hat{x}_{k}, u_{k}\right)$ is given in terms of a coupled matrix Riccati equation as follows:

$$
K_{k}\left(\hat{x}_{k}, u_{k}\right)=L_{k} \Omega_{k}\left(\hat{x}_{k}, u_{k}\right)^{-1},
$$

with

$$
\begin{aligned}
& L_{k}=A_{k} \Sigma_{k} C_{k}^{\prime}, \\
& \Omega_{k}\left(\hat{x}_{k}, u_{k}\right)=C_{k} \Sigma_{k} C_{k}^{\prime}+R_{k}+\Delta C_{k}\left(\Sigma_{k}+\hat{x}_{k} \hat{x}_{k}^{\prime}\right) \Delta C_{k}^{\prime},
\end{aligned}
$$


and

$$
\begin{aligned}
\Sigma_{k+1}= & A_{k} \Sigma_{k} A_{k}^{\prime}-L_{k} \Omega\left(\hat{x}_{k}, u_{k}\right)^{-1} L_{k}^{\prime}+Q_{k} \\
& +\Delta A_{k}\left(\Sigma_{k}+\hat{x}_{k} \hat{x}_{k}^{\prime}\right) \Delta A_{k}^{\prime}+\Delta B_{k} u_{k} u_{k}^{\prime} \Delta B_{k}^{\prime}, \\
\Sigma_{0}= & E\left[x_{0} x_{0}^{\prime}\right] .
\end{aligned}
$$

Here $\hat{x}_{k}$ is the best linear estimate conditioned on $\mathscr{Y}_{k-1}$, the $\sigma$-algebra generated by $y_{0}, \ldots, y_{k-1}$, where best is in a minimum error variance sense. The associated conditional error covariance is

$\Sigma_{k}=E\left[\left(x_{k}-\hat{x}_{k}\right)\left(x_{k}-\hat{x}_{k}\right)^{\prime} \mid \mathscr{Y}_{k-1}\right]$.

In the derivation of Eqs. (24) and (28), the Projection Theorem is used, which tells us that

$E\left[v_{k} \mid \mathscr{Y}_{k-1}\right]=0, \quad E\left[\hat{x}_{k}\left(x_{k}-\hat{x}_{k}\right)^{\prime} \mid \mathscr{Y}_{k-1}\right]=0$.

Notice that the dependence of the noise on states and controls in our model (23) leads to an error covariance which depends on the past measurements (and controls), and in turn leads to a filter gain $K_{k}(\cdot, \cdot)$ which is dependent on the past measurements (and controls). Now this dependency of $K_{k}(\cdot, \cdot)$ on $\hat{x}_{k}, u_{k}$ is by no means affine, but in order to proceed to a control law based on the LQR theory of Section 2, we must linearize $K_{k}(\cdot, \cdot)$ in $\hat{x}_{k}$ and $u_{k}$.

A filter bilinear in the innovations: Consider a linearization of $K_{k}(\cdot, \cdot)$, via a Taylor expansion, for simplicity in the $p=1$ case

$K_{k}\left(\hat{x}_{k}, u_{k}\right)=K_{k}+K_{k}^{x} \hat{x}_{k}+K_{k}^{u} u_{k}+\mathrm{o}\left(\left\|\hat{x}_{k}\right\|,\left\|u_{k}\right\|\right)$.

Neglecting the quadratic and higher-order terms in $\hat{x}_{k}, u_{k}$ leads to an approximate filter

$$
\begin{aligned}
\hat{x}_{k+1} & \approx A_{k} \hat{x}_{k}+B_{k} u_{k}+\left(K_{k}+K_{k}^{x} \hat{x}_{k}+K_{k}^{u} u_{k}\right) v_{k} \\
& =\left(A_{k}+K_{k}^{x} v_{k}\right) \hat{x}_{k}+\left(B_{k}+K_{k}^{u} v_{k}\right) u_{k}+K_{k} v_{k} .
\end{aligned}
$$

\section{State estimate feedback}

The approach taken in an LQG control design is taken here, namely to consider the state estimator (24) (or in our case the approximation (30)) as a state space signal model with state $\hat{x}_{k}$, and to re-organize the control performance index $J_{T}$ of Eq. (2) in terms of $\hat{x}_{k}$, rather than $x_{k}$. Noting Eqs. (24) and (28) we have a re-organization of $J_{T}$ as

$J_{T}=\sum_{k=0}^{T-1}\left[\hat{x}_{k}^{\prime} Q_{k}^{c} \hat{x}_{k}+u_{k}^{\prime} R_{k+1}^{c} u_{k}+\operatorname{tr}\left(Q_{k}^{c} \Sigma_{k}\right)\right]$.

Actually, $\Sigma_{k}$ is perhaps best written as $\Sigma_{k}\left(\hat{x}_{k} \hat{x}_{k}^{\prime}, u_{k} u_{k}^{\prime}\right)$ since it is dependent on $\hat{x}_{k} \hat{x}_{k}^{\prime}$ and $u_{k} u_{k}^{\prime}$. Now a Taylor series expansion leads to

$\Sigma_{k} \approx \Sigma_{k}^{0}+\Sigma_{k}^{x} \hat{x}_{k} \hat{x}_{k}^{\prime}+\Sigma_{k}^{u} u_{k} u_{k}^{\prime}$,

being linear in $\hat{x}_{k} \hat{x}_{k}^{\prime}$ and $u_{k} u_{k}^{\prime}$. Thus Eq. (31) under Eq. (32) becomes

$$
\begin{gathered}
J_{T} \approx \sum_{k=0}^{T-1}\left[\hat{x}_{k}^{\prime}\left(Q_{k}^{c}+\Sigma_{k}^{x}\right) \hat{x}_{k}+u_{k}^{\prime}\left(R_{k+1}^{c}+\Sigma_{k}^{u}\right) u_{k}\right. \\
\left.+\operatorname{tr}\left(Q_{k}^{c} \Sigma_{k}^{0}\right)\right] .
\end{gathered}
$$

Now the optimization of Eq. (33) under Eq. (30) can be tackled using the optimal LQR results of Section 2 with $w^{A}=w^{B}=w$. Thus,

$u_{k}^{\mathrm{opt}} \approx K_{k}^{c} \hat{x}_{k}+b_{k}$,

where $\hat{x}_{k}$ is derived from the filter (30). Also $K_{k}^{c}$ are derived from an approximate specialization of Eqs. (3) and (4) in which $\Delta A_{k}=K_{k}^{x}, \Delta B_{k}=K_{k}^{u}$. The term $b_{k}$ is derived by solving the algebraic equations (13) in turn for $b_{T}, b_{T-1}, \ldots, b_{0}$ being backward recursions.

\section{Conclusion}

The LQG approach to partially observed stochastic models leads to useful suboptimal state estimate linear feedback controllers when the models are bilinear in the noise and a linearization of certain equations is analysed.

\section{Acknowledgements}

The authors wish to acknowledge the funding of the activities of the Cooperative Research Centre for Robust and Adaptive Systems by the Australian Commonwealth Government under the Cooperative Research Centre Program, and the RGC Earmarked Grant CUHK 4125/97E. 


\section{References}

[1] B.D.O. Anderson, J.B. Moore, Optimal Filtering, Prentice-Hall, Englewood Cliffs, NJ, 1978.

[2] B.D.O. Anderson, J.B. Moore, Optimal Control: Linear Quadratic Methods, Prentice-Hall, Englewood Cliffs, NJ, 1989.

[3] S.P. Chen, X.J. Li, X.Y. Zhou, Stochastic linear quadratic regulators with indefinite control weight costs, SIAM J. Control Optim. 36 (1998) 1685-1702.
[4] M.H.A. Davis, Linear Estimation and Stochastic Control, Chapman \& Hall, London, 1977.

[5] R.J. Elliott, L. Aggoun, J.B. Moore, Hidden Markov Models: Estimation and Control, Springer, Berlin, 1995.

[6] A.E.B. Lim, X.Y. Zhou, Optimal stochastic LQR control with integral quadratic constraints and indefinite control weights, IEEE Trans. Automat. Control, to appear. 